\title{
ANDES

www.scielo.cl

\section{Hemiplejia Alternante de la Infancia asociada a variante patogénica del gen ATP1A3}

\author{
Alternating Hemiplegia of Childhood associated with a pathogenic variant of the \\ ATP1A3 gene
}

\author{
Francisca Sandoval $^{\oplus a}$, Francisca López ${ }^{\mathrm{a}}$
}

aHospital Dr. Exequiel González Cortés. Santiago, Chile

Recibido: 30 de julio de 2021; Aceptado: 23 de agosto de 2021

¿Qué se sabe del tema que trata este estudio?

La hemiplejia alternante de la infancia es una enfermedad de etiología genética, poco conocida e infrecuente. Se manifiesta de manera temprana en el lactante con posturas distónicas, movimientos oculares anormales y trastornos del movimiento. Antes de los 18 meses de vida los pacientes presentan episodios de hemiplejia alternantes en lateralidad, que pueden durar varios minutos a días, y que característicamente ceden durante el sueño. Los pacientes cursan con retraso en el neurodesarrollo y pueden presentar otras enfermedades neurológicas asociadas. El único tratamiento actual es la flunarizina.

\begin{abstract}
¿Qué aporta este estudio a lo ya conocido?
Reportamos el primer caso clínico de hemiplejia alterante de la infancia publicado en Chile, que cumple con criterios diagnósticos clínicos típicos y que cuenta con confirmación de mutación patogénica del gen ATP1A3. Además, presentamos videos de la clínica de la paciente presentada, útiles como recurso académico para profesionales de la salud que atienden en pediatría.
\end{abstract}

\section{Resumen}

La hemiplejia alternante de la infancia (HAI) es una enfermedad rara caracterizada por episodios de hemi/cuadriplejia, posturas distónicas, movimientos oculares anormales y trastornos del movimiento. Las mutaciones del gen ATP1A3 son las más frecuentemente asociadas a HAI. Objetivo: Exponer un caso clínico de HAI, en el que el estudio genético y la observación de videos caseros fueron de gran utilidad diagnóstica. Caso Clínico: Paciente de sexo femenino, quien a los 3 meses de vida presentó varios episodios de posturas distónicas, clonías de extremidades, versión cefálica y desviación lateral de la mirada, de varios minutos de duración. Inicialmente se diagnosticó epilepsia y se inició tratamiento con levetiracetam, sin mejoría. Se realizó EEG y RM cerebral que resultaron normales. Con esto, se descartó epilepsia y se sospechó distonía transitoria del lactante. Sin embargo, los eventos se hicieron más frecuentes, de mayor duración y característicamente cedían durante el sueño.
Palabras clave: Hemiplejia Alternante; HAI; Gen ATP1A3; Epilepsia; Flunarizina
Correspondencia:

Francisca López

franlopezavaria@gmail.com
Editado por:

Carolina Heresi Venegas 
Los familiares aportaron videos caseros aclaratorios de los eventos. A los 6 meses de vida cursó con hemiparesia transitoria alternante. El panel genético de distonías evidenció variante patogénica del gen ATP1A3, confirmando diagnóstico de HAI. Se inició tratamiento con flunarizina con buena respuesta clínica a los 12 meses de seguimiento. Conclusiones: El diagnóstico de HAI es complejo y se confunde frecuentemente con epilepsia. Es importante realizar correctamente el diagnóstico diferencial, incluyendo anamnesis, exámenes como EEG y la minuciosa observación de los eventos clínicos, que con el actual acceso a tecnología audiovisual se hace más asertivo. El análisis genético constituye una gran herramienta diagnóstica que realizado a tiempo evita otros estudios y terapias innecesarias.

\section{Abstract}

Alternating hemiplegia of childhood (AHC), is a rare disease characterized by episodes of hemi/quadriplegia, dystonic postures, abnormal eye movements, and movement disorders. ATP1A3 gene mutations are the most frequently associated with AHC. Objective: To present a clinical case of AHC, where genetic study and the observation of home videos were of great diagnostic utility. Clinical Case: Female patient who at 3 months of age presented with several episodes of dystonic postures, clonic movements of extremities, cephalic version, and lateral gaze deviation lasting several minutes. Epilepsy was diagnosed and levetiracetam was administrated, without improvement. EEG and brain MRI were performed, with normal results. Therefore, epilepsy was ruled out and transient dystonia of infancy was suspected, however, the events became more frequent, longer in duration, and characteristically subsided during sleep. Family members provided home videos that clarified the events. At 6 months of age, the patient presented with alternating hemiparesis. Dystonia genetic panel showed a pathogenic variant of the ATP1A3 gene, confirming the diagnosis. Flunarizine treatment was initiated with a good clinical response at 12 months of follow-up. Conclusions: The diagnosis of AHC is complex and is frequently confused with epilepsy, so it is important to correctly perform the differential diagnosis, including anamnesis, tests such as EEG, and careful observation of clinical events that, with the current access to audiovisual technology, becomes more accurate. The genetic analysis is a great diagnostic tool that, when performed in time, avoids other unnecessary tests and therapies.

\section{Keywords:}

Alternating

Hemiplegia;

$\mathrm{AHC}$;

ATP1A3 Gene;

Epilepsy;

Flunarizine

\section{Introducción}

La hemiplejia alternante de la infancia (HAI), es una enfermedad muy poco frecuente, de inicio en el periodo de lactante, con una incidencia aproximada de $1 / 1.000 .000$ recién nacidos sanos ${ }^{1}$. Sin embargo, se sospecha que esta cifra está subestimada, tanto por falta de conocimiento de la comunidad médica de esta patología como por la ausencia de exámenes confirmatorios previos a la aparición de estudios genéticos².

Fue descrita por primera vez en 1971 por Verret y Steele quienes la identificaban como una variante del cuadro más conocido y común de migraña hemipléjica, luego fue identificada como una entidad clínica distinta ${ }^{3}$. A pesar de varios reportes mundiales de casos, este cuadro sigue siendo de muy difícil caracterización y categorización dentro de la patología neuropediátrica. Con la intención de unificar diagnósticos, posteriormente fueron descritos criterios para su diagnóstico clínico ${ }^{4,5}$ (tabla 1 ).

La fisiopatología de la HAI es aún desconocida, los casos de HAI son mayoritariamente esporádicos, y en la última década su etiología se ha ido clarificando. Se han detectado varias mutaciones de novo asociadas, siendo las más comunes las del gen ATP1A3, mutaciones en otros genes como el CCACN1A y el ATP1A2 también han sido reportadas, las que son frecuentemente asociadas a migraña hemipléjica familiar ${ }^{1,6,7,8,9,10}$.

El gen ATP1A3, se encuentra en el cromosoma 19 q13 y codifica para la subunidad a de la bomba $\mathrm{Na}^{+} /$ $\mathrm{K}^{+}$ATPasa. Esta es una de las encargadas de la mantención de los gradientes electroquímicos en las membranas celulares excitables ${ }^{10,11}$. La subunidad a de la $\mathrm{Na}^{+}$/ $\mathrm{K}^{+}$ATPasa se expresa exclusivamente en neuronas y células de músculo cardiaco ${ }^{11,12}$.

\section{Objetivo}

Exponer un caso clínico de HAI, en el que el estudio genético y la observación de videos caseros fueron de gran utilidad diagnóstica. Reportamos el primer caso clínico de HAI publicado en Chile, que cumple con criterios diagnósticos clínicos típicos de HAI y que cuenta con confirmación de mutación patogénica del gen ATP1A3. Además, presentamos videos de la clínica de la paciente, aportados por sus tutores, para ser difundidos a la comunidad médica como recurso académico en conjunto con esta publicación. 


\section{Caso Clínico}

Lactante de sexo femenino, sin antecedentes patológicos perinatales, sana y con desarrollo psicomotor adecuado. A los 3 meses de vida inició episodios paroxísticos de posturas distónicas y clonías de las cuatro extremidades, asociadas en ocasiones a versión cefálica y desviación lateral de la mirada, de varios minutos de duración y aparente desconexión del medio, según el relato de los padres. Se realizó estudio con electroencefalograma (EEG) y tomografía computada (TC) cerebral, ambos con resultados normales. Se diagnosticó inicialmente epilepsia del lactante e inició tratamiento con fármaco antiepiléptico (FAE) levetiracetam. Paciente evolucionó sin mejoría clínica, por lo que a los 5 meses consultó con especialista en epilepsia y se complementó estudio con EEG prolongado de 2 horas y Resonancia Magnética (RM) cerebral, ambos normales (figura 1). Posteriormente se descartó epilepsia y se sospechó distonía transitoria del lactante (DTL), se indicó suspensión de levetiracetam.

Posterior a la suspensión del levetiracetam, la paciente evoluciona con aumento de la frecuencia (más de 5 al día) y de la duración (algunos de horas de duración) de los eventos, presentándose agrupados en lapsos de 2 a 3 días, con períodos de días libres de síntomas. Característicamente estas posturas anormales y alteraciones oculomotoras cedían durante el sueño y reincidían unos minutos después del despertar. Los padres de la paciente aportaron varios videos de los eventos clínicos (Código de escaneo Video 1).
Tabla 1. Criterios Diagnósticos de Hemiplejia Alternante de la Infancia ${ }^{4,5}$

- Edad de inicio de clínica < 18 meses

- Episodios de hemiplejia que alternan lateralidad de ambos hemicuerpos, de minutos a días de duración

- Episodios de hemiplejia bilateral/ cuadriplejia

- Otros trastornos paroxísticos:

- Posturas distónicas

- Movimientos oculares anormales

- Otros trastornos del movimiento

- Desaparición de síntomas durante el sueño y reaparición a los 10 minutos tras el despertar

- Alteraciones en el neurodesarrollo, dificultades cognitivas y de aprendizaje, y/o secuelas neurológicas de tipo ataxia, distonía y coreoatetosis

Exclusión de otros trastornos que causen crisis de hemiplejia recurrente

Debido a evolución no concordante con diagnóstico de DTL, se decidió ampliar el estudio hospitalizando a la paciente a los 6 meses de vida. Se realizó estudio con perfil de aminoácidos y acilcarnitinas mediante espectrometría de masas en tandem y angioRM cerebral, ambos con resultados normales. Durante estadía hospitalaria presentó un episodio de hemiparesia derecha que duró 6 horas con recuperación completa. Un día después presentó recurrencia ipsilateral de la hemiparesia esta vez de casi 12 horas duración, nuevamente autolimitada. Luego de 24 horas de mantenerse

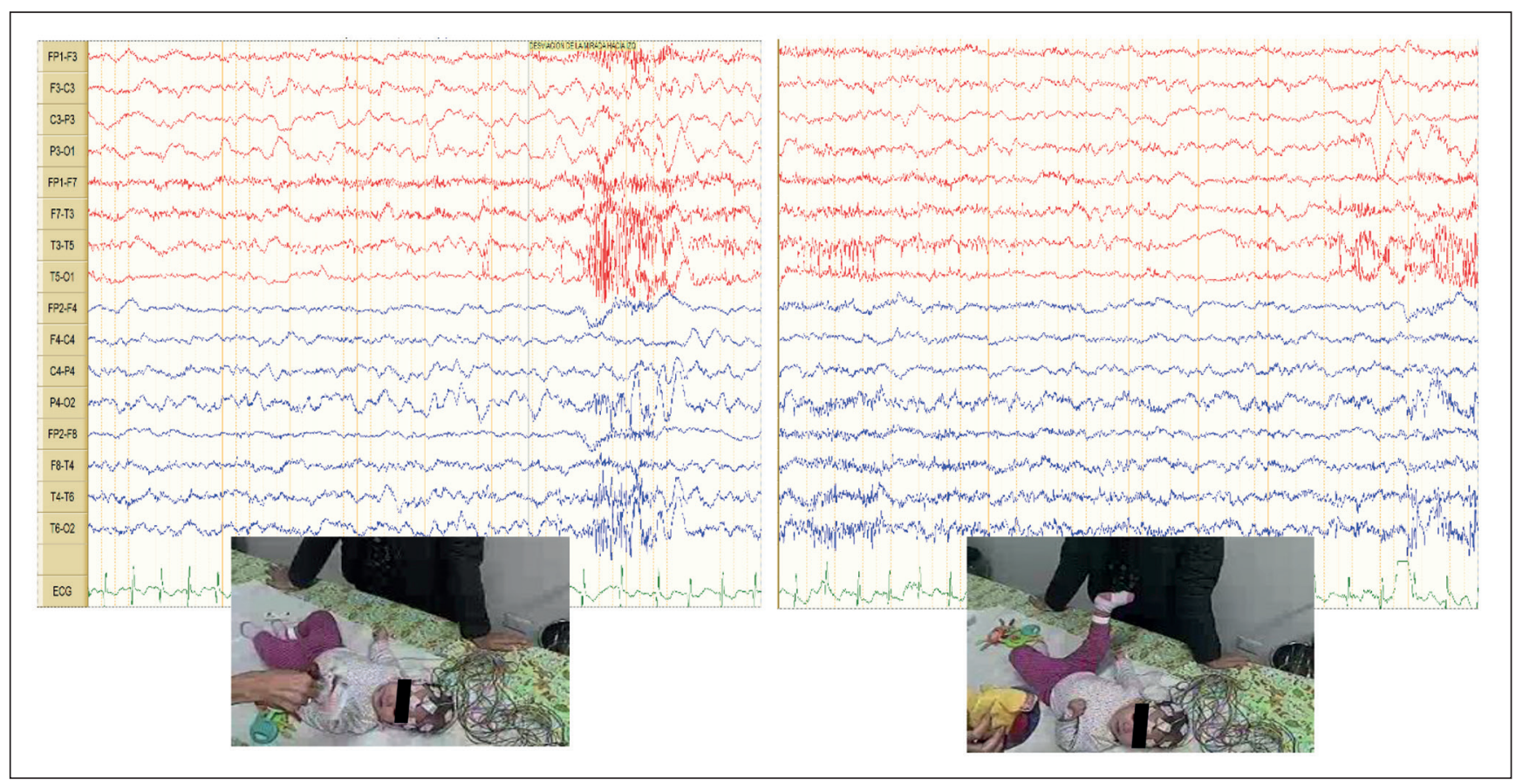

Figura 1. EEG de vigilia de la paciente, cursando con evento clínico característico sin correlato eléctrico epiléptico. 


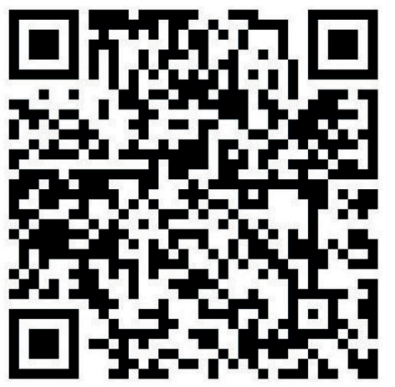

VIDEO 1

Video 1. Episodios prolongados de distonía asimétrica de 4 extremidades, clonías y versión cefálica presentados por la paciente a los 5 meses de edad. Nota: Para escanear códigos de video debe enfocar la imagen con la cámara de su Smartphone y abrir el enlace que se despliega. En ocasiones tendrá que instalar una aplicación que sea lector de códigos QR.

asintomática la paciente presentó hemiparesia del lado contralateral (Código de escaneo Video 2).

Se planteó el diagnóstico de HAI, por lo que se realizó panel genético de distonías que evidenció variante patogénica, c.2263G > T (pGly755Cys) del gen ATP1A3. Posteriormente se inició tratamiento con flunarizina a dosis de $10 \mathrm{mg}$ día, con disminución considerable de frecuencia, duración e intensidad de los episodios paroxísticos. Como parte de su tratamiento, la paciente también recibió de manera regular sesiones de rehabilitación motora y estimulación integral desde los 7 meses de vida.

La paciente evolucionó con retraso del desarrollo psicomotor (RDSM), dijo sus primeras palabras con sentido a los 16 meses de vida, y a los 18 meses logró bipedestación y gateo, no así marcha lateral.

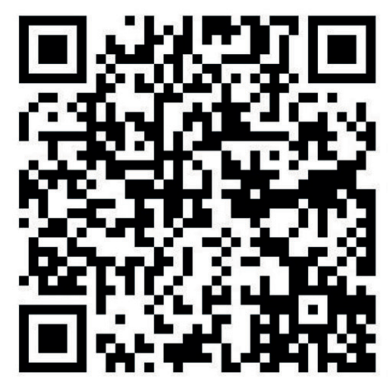

VIDEO 2

Video 2. Episodios de hemiplejia que alternan en lateralidad presentados por la paciente a los 6 meses de edad.

Nota: Para escanear códigos de video debe enfocar la imagen con la cámara de su Smartphone y abrir el enlace que se despliega. En ocasiones tendrá que instalar una aplicación que sea lector de códigos QR.
En el control ambulatorio a la edad de 18 meses la paciente continuaba presentando eventos aislados de hemiplejia y de distonía (en promedio 1 al mes, de horas de evolución) todos en vigilia. Durante 12 meses de seguimiento y de tratamiento con flunarizina, la paciente no manifestó eventos adversos asociados al uso del medicamento. Tampoco presentó crisis epilépticas durante el periodo de observación.

\section{Discusión}

La HAI es una enfermedad rara de difícil identificación y diagnóstico. Tiene una baja incidencia, y es bastante desconocida por equipos de salud. Las manifestaciones habituales y más tempranas de ésta son los episodios distónicos y movimientos oculares anormales. Posteriormente se agregan los eventos de hemiplejia característicos ${ }^{1,3,4,5}$. El diagnóstico diferencial más relevante es con epilepsia del lactante, que puede entorpecer y retrasar el diagnóstico de HAI hasta la aparición de las crisis de hemiplejia recurrentes que alternan lateralidad de ambos hemicuerpos ${ }^{13,14}$.

$\mathrm{Al}$ igual que lo descrito en la literatura, nuestra paciente inició precozmente con eventos paroxísticos de distonías y movimientos oculares anormales, por lo que recibió tratamiento antiepiléptico con pobre respuesta a éste. La hemiplejia alternante se agregó 3 meses después del inicio de los síntomas. En ella, el diagnóstico de epilepsia posteriormente fue descartado sin gran dificultad luego de revisar los videos de los eventos aportados por padres (Códigos de escaneo video 1 y 2) y EEGs consistentemente normales (figura 1). Es semiológicamente relevante en este caso, que nuestra paciente presentaba los eventos sólo en vigilia, y que estos cedían durante el sueño y reincidían al despertar, cumpliendo con los criterios de diagnóstico clínico actualmente vigentes ${ }^{5}$ (tabla 1 ).

Existen múltiples diagnósticos diferenciales de HAI que se manifiestan como distonía en el lactante, como la DTL que tiene un pronóstico benigno. Otros diagnósticos pueden presentarse con hemiparesia como punto cardinal, incluyendo migraña hemipléjica familiar, y algunas enfermedades neurodegenerativas como lo son las enfermedades mitocondriales. Dentro de estas, destaca el síndrome con encefalopatía mitocondrial, acidosis láctica y episodios parecidos a accidente cerebrovascular (MELAS), que tiene mal pronóstico ${ }^{13}$ (tabla 2).

La paciente presentada en este caso clínico cuenta con confirmación diagnóstica mediante test genético que evidencia la variante patogénica c.2263G > T (pGly755Cys) del gen ATP1A3, las mutaciones de este gen son las más frecuentemente reportadas en pacientes que presentan esta enfermedad ${ }^{6,8,9,10}$. Otras mutaciones conocidas son: c.2443G > A (E815K), c.2401G 
Tabla 2. Diagnósticos diferenciales de Hemiplejia Alternante de la Infancia.

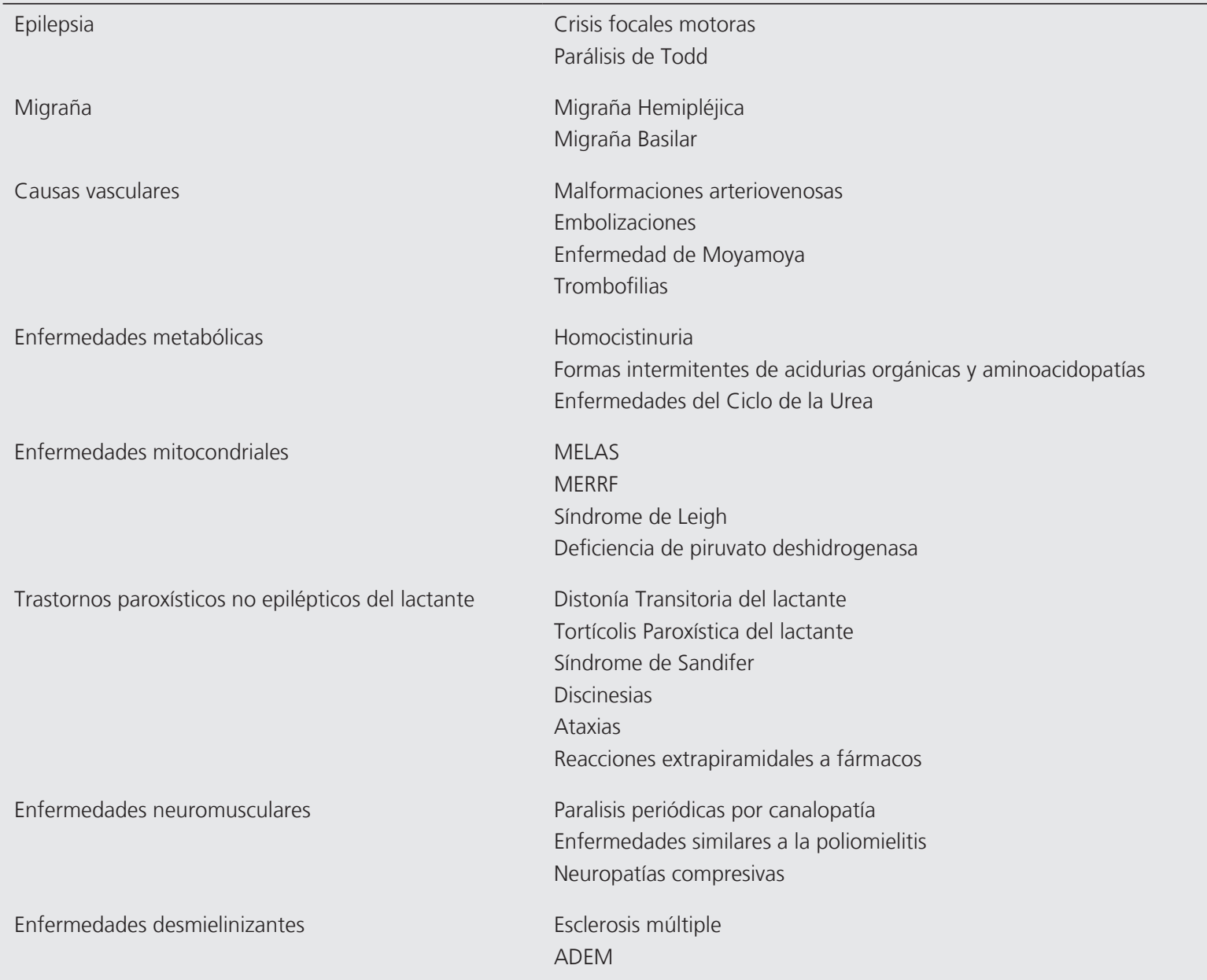

MELAS: síndrome con encefalopatía mitocondrial, acidosis láctica y episodios parecidos a accidente cerebrovascular. MERRF: Epilepsia mioclónica con fibras rojas rasgadas ADEM: Encefalomielitis Aguda Diseminada.

$>$ A $(\mathrm{D} 801 \mathrm{~N})$, c.2839G > A (G947R) y c.2443G > A (C927Y). También variantes de los genes CCACN1A y el ATP1A2 han sido reportadas en pacientes con HAI, éstas mayoritariamente se asocian a migraña hemipléjica familiar $78,9,15,16,17$.

En cuanto al manejo de la HAI, existe vasta evidencia sobre el uso de flunarizina, demostrando que disminuye de manera considerable los eventos hemipléjicos y distónicos de pacientes con HAI. Además, existe actualmente evidencia en desarrollo del uso de ATP oral para manejo de esta patología ${ }^{2,18,19,20}$. Nuestra paciente tuvo buena respuesta al tratamiento con flunarizina, único medicamento actualmente disponible a nivel local para esta patología, con disminución clara en frecuencia y gravedad de sus episodios tanto hemipléjicos como distónicos.

Dentro de las alteraciones del neurodesarrollo descritas en pacientes con HAI, el RDSM es el más fre- cuente $^{1,3}$. Nuestra paciente, al igual que lo descrito desde el primer reporte de HAI por Verret y Steele ${ }^{3}$, ha evolucionado con RDSM, incluso a pesar de haber tenido buena respuesta al tratamiento farmacológico e intervención temprana con fisioterapia y estimulación integral.

Según lo reportado a nivel mundial un bajo número de pacientes puede presentar posteriormente en su evolución epilepsia, que en general es de fácil manejo $^{6,14,15}$. La paciente presentada, hasta la edad de 18 meses no ha presentado nunca crisis epilépticas.

\section{Conclusiones}

El diagnóstico de HAI es complejo y se confunde muy frecuentemente con epilepsia, por eso es de especial relevancia realizar correctamente el diagnóstico diferencial, realizando una detallada anamnesis, com- 
plementada con exámenes como EEG y la minuciosa observación de los eventos clínicos, que ahora con el fácil acceso a tecnología audiovisual se hace más asertivo.

Es necesario hacer conocida esta enfermedad en la comunidad médica que atiende a lactantes, con la intención de generar diagnósticos tempranos y precisos. Consideramos que el análisis genético en este caso y en los venideros, constituye una gran herramienta diagnóstica, que realizado a tiempo evita otros estudios y terapias innecesarias, como es el uso de FAEs.

\section{Responsabilidades Éticas}

Protección de personas y animales: Los autores declaran que los procedimientos seguidos se conformaron a las normas éticas del comité de experimentación humana responsable y de acuerdo con la Asociación Médica Mundial y la Declaración de Helsinki.

Confidencialidad de los datos: Los autores declaran que han seguido los protocolos de su centro de trabajo sobre la publicación de datos de pacientes.

Derecho a la privacidad y consentimiento informado: Los autores han obtenido el consentimiento informado de los padres (tutores) de la paciente y/o sujetos referidos en el artículo. Este documento obra en poder del autor de correspondencia

\section{Conflicto de intereses}

Los autores declaran no tener conflicto de intereses.

\section{Referencias}

1. Hoei-Hansen CE, Dali CÍ CE, Lyngbye TJ, et al. Alternating hemiplegia of childhood in Denmark: clinical manifestations and ATP1A3 mutation status. Eur J Paediatr Neurol. 2014;18:50-4.

2. Neville BG, Ninan M. The treatment and management of alternating hemiplegia of childhood. Dev Med Child Neurol. 2007;49:777-80.

3. Verret S, Steele JC. Alternating hemiplegia in childhood: a report of eight patients with complicated migraine beginning in infancy. Pediatrics 1971;47:675-80.

4. Bourgeois M, Aicardi J, Goutieres F. Alternating hemiplegia of childhood. J. Pediatr. 1993;122:673-9.

5. Panagiotakaki E, Gobbi G, Neville B, et al. Evidence of a non-progressive course of alternating hemiplegia of childhood: study of a large cohort of children and adults. Brain. 2010;133:3598-610.

6. Panagiotakaki E, De Grandis E, Stagnaro $\mathrm{M}$, et al. Clinical profile of patients with ATP1A3 mutations in Alternating Hemiplegia of Childhood-a study of 155 patients. Orphanet J Rare Dis. 2015;10:123

7. Fons C, Campistol J, Narbona J, et al. Hemiplejía alternante de la infancia en la población española. Estudio de una serie de 17 pacientes. Med Clin (Barc). 2008;130: 577-9.
8. Ishii A, Saito Y, Mitsui J, et al. Identification of ATP1A3 mutations by exome sequencing as the cause of alternating hemiplegia of childhood in Japanese patients. PloS One. 2013;8:e56120.

9. Rosewich $\mathrm{H}$, Thiele $\mathrm{H}$, Ohlenbusch A, et al. Heterozygous de-novo mutations in ATP1A3 in patients with alternating hemiplegia of childhood: a whole-exome sequencing gene-identification study. Lancet Neurol. 2012;11(9):764-73.

10. Heinzen EL, Swoboda KJ, Hitomi Y, et al. De novo mutations in ATP1A3 cause alternating hemiplegia of childhood. Nat Genet. 2012;44:1030-4

11. Lingrel JB, Williams MT, Vorhees CV, et al. Na, K- ATPase and the role of alpha isoforms in behaviour. J Bioenerg Biomembr. 2007;39:385-9.

12. Blanco-Arias P, Einholm AP, Mamsa H, et al. A C-terminal mutation of ATP1A3 underscores the crucial role of sodium affinity in the pathophysiology of rapidonset dystonia-parkinsonism. Hum Mol Genet. 2009;18(13):2370-7.

13. Ramírez-Zamora M, Ortez- González CI. Hemiplejía alternante de la infancia. Primer caso clínico descrito en El Salvador. Rev Neurol. 2013;57:212-6.

14. Guevara-Campos J, González-de Guevara L, Urbáez-Cano J, et al. Hemiplejía alternante de la infancia tratada como epilepsia. Dos nuevos casos [Alternating hemiplegia of childhood treated as epilepsy. Two new cases]. Rev Neurol. 2005;40(6):351-3.

15. Pavlidis E, Uldall P, Gøbel Madsen C, et al. Alternating hemiplegia of childhood and a pathogenic variant of ATP1A3: a case report and pathophysiological considerations. Epileptic Disord. 2017;19(2):226-30.

16. Sasaki M, Ishii A, Saito Y, et al. Genotypephenotype correlations in alternating hemiplegia of childhood. Neurology. 2014;82(6):482-90.

17. Sweney MT, Newcomb TM, Swoboda KJ. The expanding spectrum of neurological phenotypes in children with ATP1A3 mutations, Alternating Hemiplegia of Childhood, Rapid-onset Dystonia-Parkinsonism, CAPOS and beyond. Pediatr Neurol. 2015;52(1):56-64.

18. Pisciotta L, Gherzi M, Stagnaro M, et al. Alternating Hemiplegia of Childhood: Pharmacological treatment of 30 Italian patients. Brain Dev. 2017;39(6):521-8

19. Ju J, Hirose S, Shi XY, et al. Treatment with Oral ATP decreases alternating hemiplegia of childhood with de novo ATP1A3 Mutation. Orphanet J Rare Dis. 2016;11(1):55

20. Samanta D. Management of Alternating Hemiplegia of Childhood: A Review. Pediatr Neurol. 2020;103:12-20. 